\title{
High Heat Flux Testing of Doped and Coated Graphite Using High-Intensity Pulsed Ion Beam
}

\author{
X.P. Zhu, C. LiU AND M.K. LeI* \\ Surface Engineering Laboratory, School of Materials Science and Engineering \\ Dalian University of Technology, Dalian 116024, China
}

\begin{abstract}
A high-intensity pulsed ion beam (HIPIB) technique is applied to heat flux testing of plasma facing materials for fusion experiment. The HIPIB is generated at a relatively stable power density up to $10^{8} \mathrm{~W} / \mathrm{cm}^{2}$, which covers a heat flux parameter of up to several hundreds $\mathrm{MW} \mathrm{m} \mathrm{m}^{-2} \mathrm{~s}^{1 / 2}$. Surface morphology and weight loss are examined for doped and coated graphite with HIPIB exposure of $280 \mathrm{MW} \mathrm{m}{ }^{-2} \mathrm{~s}^{1 / 2}$, being of the same order of thermal loads during off-normal events in future fusion reactors. The work demonstrates a first example utilizing the HIPIB technique to study thermal response of plasma facing materials under fusion relevant thermal loads.
\end{abstract}

PACS numbers: 28.52.Fa, 52.59.Mv, 81.05.Uw

\section{Introduction}

Initiated by International Thermonuclear Experimental Reactor (ITER) project, extensive efforts are contributed to development of advanced plasma facing materials (PFM) $[1,2]$. Existing fusion devices hardly provide the conditions to study the performance of PFMs under the ITER specific thermal loads. High heat flux (HHF) test facilities based on intense electron and ion beams are thus utilized to simulate the high thermal loads not including neutron effect for assessing different material designs and their performances. There is still a challenge to reliably simulate the short transient events depositing energy of up to several tens of MJ $\mathrm{m}^{-2}$ onto PFMs in microseconds to seconds scale. The HHF testing data should be carefully interpreted as obtained from different test facilities. Improvements in present HHF testing approaches are necessary for a reliable and efficient material assessment. Electron beams have small spots of 1-10 $\mathrm{mm}$ diameter and the incident energy is notably reflected for high- $Z$ materials; large-area ion beams could be produced up to several tens of centimetres diameter but with limited peak power density [2]. In addition, ion beam power density may fluctuate as large as $50 \%$ [3]. In our laboratory, high-intensity pulsed ion beam (HIPIB) of power density up to $10^{8} \mathrm{~W} / \mathrm{cm}^{2}$ is achieved with a beam fluctuation controlled within 20\% [4], which allows us for a better reproducibility of PFMs HHF testing. A heat flux parameter $P t^{1 / 2}$, the product of power density and the square root of the pulse duration, is used to characterize the heat flux intensity, as proposed pre-

* corresponding author; e-mail: surfeng@dlut.edu.cn viously [2]. Thermal response of carbon-based PFMs is examined for the first time using the HIPIB technique.

\section{HHF testing experiment by high-intensity pulsed ion beam}

HIPIB composed of mainly proton ions is produced in TEMP-6 HIPIB apparatus applying an improved magnetically insulated ion diode (MID) [4]. The MID cathode is powered symmetrically by two current transformers, working as one-turn "close" coil to build insulating magnetic field. The special MID design ensures HIPIB generation of more stable parameters, larger beam spot and higher power density in comparison with conventional MIDs. The focused ion beam spot is of an ellipse shape around $16 \mathrm{~cm}^{2}$ at power density of $10^{8} \mathrm{~W} / \mathrm{cm}^{2}$. Two types of carbon-based PFMs for the test are doped graphite GBST1308 $\left(1 \% \mathrm{~B}_{4} \mathrm{C}, 2.5 \% \mathrm{Si}, 7.5 \% \mathrm{Ti}\right)$ and gradient SiC coating on GBST1308 prepared by combined chemical vapour reaction and infiltration [5]. The applied HIPIB parameters are $300 \mathrm{kV}$ (accelerating voltage) and $350 \mathrm{~A} / \mathrm{cm}^{2}$ (ion current density) with a pulse duration of $75 \mathrm{~ns}$, i.e. a heat flux parameter of $280 \mathrm{MW} \mathrm{m}^{-2} \mathrm{~s}^{1 / 2}$ being of the same order of thermal loads for ITER off-normal events, and the tests are performed with 1 - or 5 -shot irradiation. The uncoated samples are mechanically polished and the coated ones are in as-prepared state. Commercial pure graphite is tested for comparison. Surface morphology of samples is examined by using a JSM-5600LV scanning electron microscope, the surface profile and roughness by a Surfcorder ET4000M profilometer, and the weight loss by a SARTORIUS BS124S microelectronic balance, respectively. 


\section{Results and discussion}

Surface morphologies of pure and doped graphite after HIPIB irradiation are presented in Fig. 1. The surfaces are partly shielded for 1-shot HHF exposure, where the image shows shielding edge with original surface on the left and the HHF exposed on the right, and obvious surface ablation is found with etching or exfoliation after exposure (Fig. 1a, b). More etching channels occur on the 5-shot exposed surfaces (Fig. 1c, d), attributable to selective ablation of grain boundary. As inferred from the ablated patterns, the doped graphite seems to have smaller and more uniformly distributed grains with a more uniform ablation.

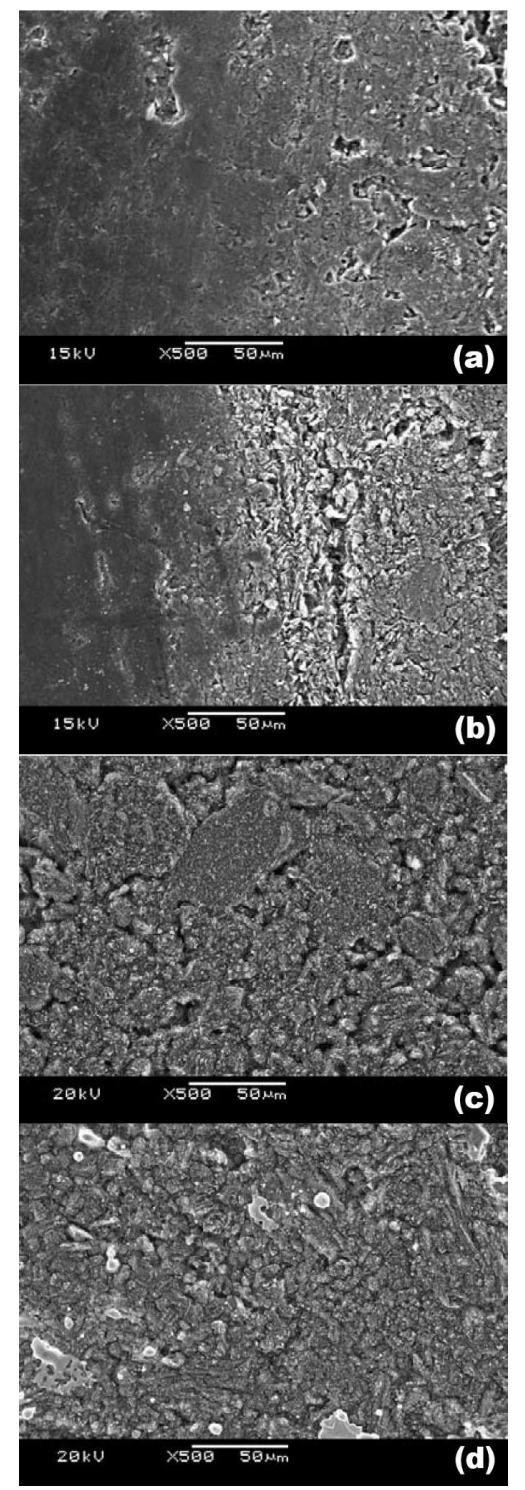

Fig. 1. Surface morphologies of (a) graphite and (b) doped graphite with original morphology under shielding on the left after 1-shot HHF exposure, and (c) graphite and (d) doped graphite after 5-shot exposure at a heat flux parameter of $280 \mathrm{MW} \mathrm{m}^{-2} \mathrm{~s}^{1 / 2}$ (scale bar: $50 \mu \mathrm{m})$.
Figure 2 shows surface morphologies of SiC coated GBST1308 before and after irradiation. The original surface presents a feature of packed particles. The morphology has little change after 1-shot HHF exposure, except for that some larger particles of about $20 \mu \mathrm{m}$ occur due to merging of small particles melted. The morphology greatly changes after 5-shot exposure, with a melted and ablated pattern of knots, losing the feature of discrete particles. The changes imply that the loosely packed particles on original coatings are preferentially removed under the repetitive melting and ablation.

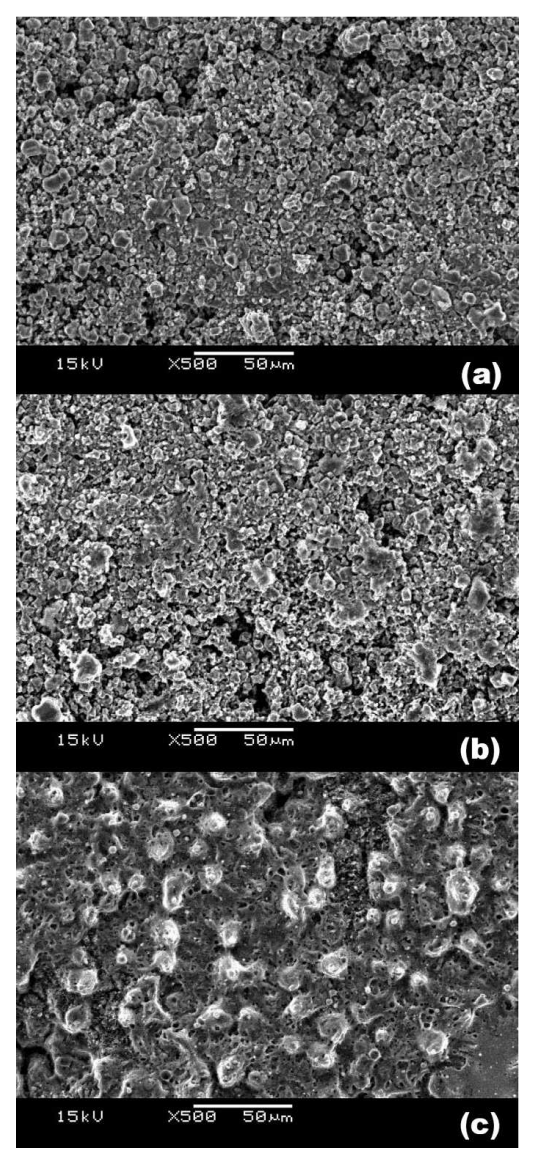

Fig. 2. Surface morphologies of SiC coatings: (a) original, (b) 1-shot HHF exposure and (c) 5-shot exposure at a heat flux parameter of $280 \mathrm{MW} \mathrm{m}^{-2} \mathrm{~s}^{1 / 2}$ (scale bar: $50 \mu \mathrm{m})$.

Surface profiles of the samples before and after 1-shot HHF exposure are shown in Fig. 3, respectively. Significant roughening is found on the bulk graphite after the HHF exposure, but the profile curve of GBST1308 has a slower slope (Fig. 3a) in accordance with a more flat ablated surface (Fig. 1). The $\mathrm{SiC}$ coating has high roughness due to its as-prepared feature, and the roughness increases slightly with more large peaks and valleys on the profile curve, relating to particle merging (Fig. $2 \mathrm{~b}$ ).

The weight loss of the samples after 5 -shot HHF exposure is summarized in Fig. 4 . The values are about $0.45,0.29$ and $0.18 \mathrm{mg} / \mathrm{cm}^{2}$ for graphite, doped graphite 


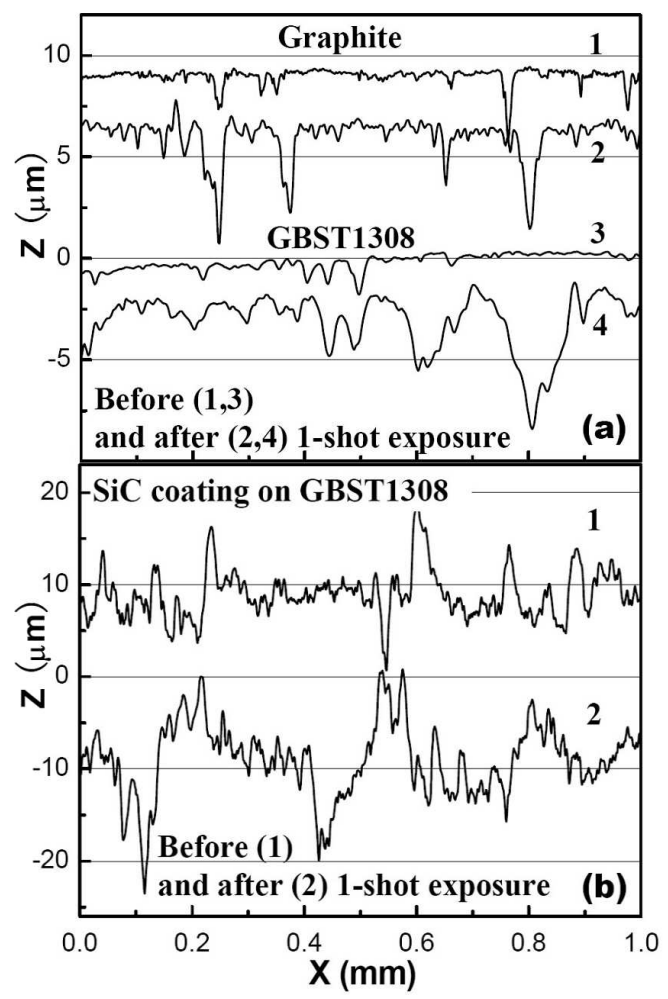

Fig. 3. Surface profiles of different samples: (a) graphite and doped graphite and (b) $\mathrm{SiC}$ coatings before and after 1-shot HHF exposure at a heat flux parameter of around $280 \mathrm{MW} \mathrm{m}^{-2} \mathrm{~s}^{1 / 2}$.

and $\mathrm{SiC}$ coating, respectively. Assuming a density of 2.1, 2.3 , and $3.0 \mathrm{~g} / \mathrm{cm}^{3}$ for graphite, doped graphite and $\mathrm{SiC}$ coating, the average erosion rate is about $2.2,1.2$, and $0.6 \mu \mathrm{m}$ on the respective samples at 5-shot HHF exposure. A two-dimensional heat transfer model with enthalpy formulation was developed to study thermal and dynamical effects under HIPIB irradiation, and the calculated ablation rate on graphite is about $0.8 \mu \mathrm{m}$ per shot at $350 \mathrm{~A} / \mathrm{cm}^{2}$ [4], i.e. a heat flux parameter identical to this study. The erosion rate of bulk material must be underestimated due to redeposition of ablated matters onto the samples. More factors should be considered for the erosion assessment of the gradient $\mathrm{SiC}$ coating due to the variations in composition and density along the ablation depth. Nevertheless, this preliminary study demonstrates a possibility of utilizing the HIPIB technique as a new HHF testing approach to PFMs development for future fusion reactor application.

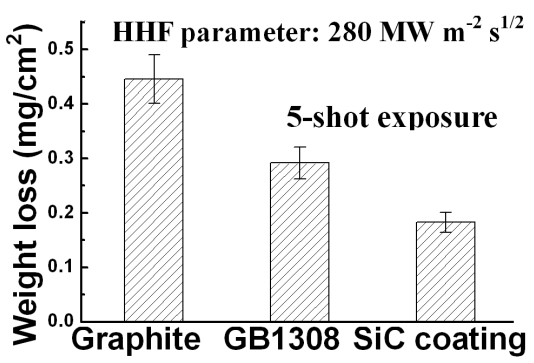

Fig. 4. Weight loss of graphite, doped graphite, and $\mathrm{SiC}$ coating on doped graphite after 5 -shot HHF exposure at a heat flux parameter of $280 \mathrm{MW} \mathrm{m}^{-2} \mathrm{~s}^{1 / 2}$.

\section{Conclusions}

A HIPIB technique is utilized to evaluate performance of plasma facing materials, simulating extremely high heat flux during plasma-materials interactions. It is demonstrated that the HIPIB technique can be used as a flexible method to assess the different material designs and their performances under high heat flux exposure as anticipated in the future fusion reactors.

\section{Acknowledgments}

The authors would like to thank Dr. J.L. Chen (Institute of Plasma Physics, Chinese Academy of Sciences) for his valuable discussion and providing the samples of PFMs. This work is supported by National Basic Research Program of China (973 Program) under No. 2008 CB717801.

\section{References}

[1] G. Federici, C.H. Skinner, J.N. Brooks, J.P. Coad, C. Grisolia, A.A. Haasz, A. Hassanein, V. Philipps, C.S. Pitcher, J. Roth, W.R. Wampler, D.G. Whyte, Nuclear Fusion 41, 1967 (2001).

[2] J. Linke, F. Escourbiac, I.V. Mazul, R. Nygren, M. Rodig, J. Schlosser, S. Suzuki, J. Nuclear Mater. 367-370, 1422 (2007).

[3] A.R. Raffray, J. Blanchard, J. Latkowski, F. Najmabadi, T. Renk, J. Sethian, S. Sharafat, L. Snead, the HAPL Team, Fusion Eng. Design 81, 1627 (2006).

[4] X.G. Han, S.M. Miao, X.P. Zhu, M.K. Lei, Appl. Surf. Sci. 253, 5425 (2007).

[5] J.L. Chen, F. Gao, J.G. Li, Q.F. Fang, J. Nuclear Mater. 363-365, 1334 (2007). 